\title{
A novel modeling approach for a capacitated $(S, T)$ inventory system with backlog under stochastic discrete demand and lead time
}

\author{
Pham Duc Tai ${ }^{a}$, Pham Phuong Ngoc Huyen ${ }^{b}$ and Jirachai Buddhakulsomsiri ${ }^{*}$
}

anternational Academy of Aviation Industry, King Mongkut's Institute of Technology Ladkrabang, Thailand

${ }^{b}$ School of Manufacturing Systems and Mechanical Engineering, Sirindhorn International Institute of Technology, Thammasat University, Thailand

\section{CH R O N I C L E}

\section{Article history:}

Received July 92020

Received in Revised Format

October 72020

Accepted October 282020

Available online

October, 282020

Keywords:

Order-up-to level policy

Periodic review

Warehouse capacity

Stochastic demand

Uncertain lead time

\begin{abstract}
A B S T R A C T
In this paper, a new period-based approach is proposed for modeling a capacitated inventory system, operating under an (S,T) policy with backlog. The system experiences stochastic discrete demand and lead time. By using the proposed method, a mathematical model is developed. The model can accurately estimate the inventory system measures of performance: the expected inventory on-hands and over-storage amount. Through a simulation experiment, the new model is compared with two other models, which are developed by using a widely used mean-based approach. The comparison is conducted based on a case study data set. The results demonstrate that the period-based model is superior to the mean-based models with respect to capturing the behaviors of the inventory system. Therefore, better inventory policy parameters can be obtained by employing the new model.
\end{abstract}

\section{Introduction}

A periodic review, order-up-to level, known as replenishment cycle, is one of the most widely studied inventory policies in the Operations Research (OR) literature. Its operational principles and basic formulations, regarded as the traditional meanbased model in this paper, have been discussed in detail by Hadley and Whitin (1963) and Silver et al. (2016). Several modifications and extensions have been made to accommodate this policy for various operational settings and constraints, including different demand distributions (Teunter et al., 2010; Nenes et al., 2010; Moharana and Sarmah, 2016), uncertain replenishment lead time (Zipkin, 1988; Keaton, 1995; Levén and Segerstedt, 2004), and limited storage capacity (Archibald, 2007). An important aspect of effectively applying this inventory policy depends on the ability to model the demand during a period and an uncertain protection interval (i.e., review period plus lead time). According to Silver et al. (2016), the normal distribution is usually a good representation for the observed demand data. However, the normal and other theoretical distributions may not be appropriate for the demand of slow-, medium-, and some fast-moving items that contain high-, moderate and low-percentage periods with no demand, i.e. intermittent demand, respectively. This is observed in many levels of a supply chain, ranging from downstream retailers to upstream distribution centers (DCs) (Singha et al., 2019). In addition, for demand during a protection interval, Silver et al. (2016) state that normal distribution would still be a valid approximation for a long enough protection interval. Unfortunately, the length of this interval in some practical situations is often kept under a few periods because of its dramatic effect on the bullwhip effect (Lee et al., 1997a,b). In addition, lead time is not always constant, e.g., a delivery may be on-time in most cycles with a small chance of being slightly delayed. As a result, the application of normal distribution for the demand and the demand during a protection interval becomes limited. The same argument can also be made for other theoretical distributions. When fitting standard distributions becomes ineffective, some researchers, including Scarf(1958), Bulinskaya (1990), Gallego (1992), Gallego and Moon (1993), and Moon and Choi (1994,

* Corresponding author

E-mail: jirachai@siit.tu.ac.th (J. Buddhakulsomsiri)

2021 Growing Science Ltd.

doi: $10.5267 / j$. ijiec.2020.10.004 
1995), have suggested a distribution-free approach, in which the optimal policy is determined by using the worst possible distribution. This approach was applied for the single-period newsboy problem (Scarf, 1958; Gallego and Moon, 1993; Moon and Choi, 1995) and continuous review system (Bulinskaya, 1990; Gallego, 1992; Moon and Choi, 1994). The applicability of the approach for the periodic review system has not been validated. As a result, modeling discrete demand during a protection interval so that an optimal policy could be determined remains a challenge.

Given the limitations of using theoretical distributions to model the demand, the traditional mean-based model would inaccurately estimate the expected inventory on-hands, a critical component in calculating the total inventory cost. Indeed, this behavior is observed when we construct a simulation model of an $(S, T)$ inventory system and compare the expected inventory on-hands with the estimate from the mean-based model. Consequently, finding optimal inventory policy based on the mean-based model's estimate would result in a sub-optimal policy. To overcome this issue, a novel approach, which does not rely on the underlying assumption of the demand distribution during a protection interval, is developed in this paper. Our model can produce an accurate estimate of the expected inventory on-hands, and, therefore, leading to an optimal policy. This paper also considers another practical issue, which is the limitation of owned warehouse space. With this limit, additional spaces at an external warehouse are rented to keep excessive inventory at a higher holding cost, called over-storage cost in this paper. Similar to the holding cost, the over-storage cost is incurred based on the expected over-storage amount within a replenishment cycle, which may be inaccurately estimated when applying the mean-based model. In this paper, the expected over-storage is determined by using the same period-based model as in the case of the expected inventory on-hands. Effectiveness of the proposed method is also demonstrated.

The periodic review, order-up-to level policy for a capacitated inventory system with backlog under study is derived from a case study DC that serves hundreds of retailers in Thailand. The proposed period-based approach is, therefore, tested with real data obtained from the case study DC to demonstrate the method's effectiveness. In summary, our paper has the following contributions:

1. We consider the inventory system, whose demand does not necessarily follow a well-known probability distribution. In addition, the characteristics of lead time observed at the case study DC is that the lead time is discrete, stochastic, and skewed to the right. Together with the characteristics of the demand, i.e. discrete, stochastic, and intermittent, the demand during a protection period is modeled by using an empirical distribution, which is simply a distribution that fits the pattern of the demand according to the observed data.

2. A period-based approach that can accurately estimate the behavior of the inventory system is proposed. Specifically, mathematical expressions for the expected inventory on-hands and over-storage are developed. With these expressions, a simple heuristic is developed to determine the optimal policy for the inventory system.

3. Our proposed model is compared with the traditional mean-based model by using a simulation experiment based on two data sets, a slow-moving item and fast-moving item, obtained from the case study DC. The results indicate that the proposed model can effectively capture the behaviors of the system, leading to a better policy than the meanbased model.

\section{Literature Review}

In our review, we classify previous and relevant studies using two factors, internal and external. Internal factors include inventory policy decisions, storage capacity constraints, and product characteristics. Inventory policy decisions deal with reviewing inventory and placing a replenishment order, i.e., time-oriented (periodic review) or quantity-oriented (continuous review with a reorder point), and determining the order quantity, i.e., fixed quantity (EOQ) or varied quantity (order-up-to level). For the storage capacity constraints, there are hard and flexible constraints. The hard constraint does not allow the inventory on-hands to exceed a capacity limit, while the flexible constraint allows over-storage by overflowing to an external incapacitated warehouse. In addition, the storage can be either dedicated for a single item or shared among multiple items. For product characteristics, an item can be either seasonal or continuously-stocked, and be either perishable or nonperishable. For external factors, we consider the characteristics of demand and lead time (deterministic and/or stochastic), and shortage (backlogged, lost sale, or partially backlogged). The deterministic component of demand may contain a constant or a function of several variables, such as price, product shelf-life, remaining stock, etc. A stochastic component may be a random variable that follows a known probability distribution or a stochastic process. Lead time may be negligible, constant, or random. Different types of shortages affect the stock level with respect to how the unmet demand is handled. Since there is a vast literature in inventory management, only the most related papers, considering non-perishable items with storage capacity, are reviewed in this paper. A comprehensive review of studies related to perishable items and limited storage can be found in Bakker et al. (2012) and Janssen et al. (2016).

Among relevant papers, both continuous review and periodic review policies are discussed. For continuous review, $(Q, R)$ is a common policy. The studies of $(Q, R)$ with a hard storage capacity constraint are Hariga (2010), Zhao et al. (2012), and Pan et al. (2014). Hariga (2010) and Pan et al. (2014) consider a single item inventory system with backlogged, stochastic demand (stationary distribution and compound Poisson, respectively), and deterministic lead time. Zhao et al. (2012) consider a similar setting for multiple items. In contrast, Lee et al. (2016) and Sana (2015) consider a flexibile capacity constraint, in which an external warehouse is rented to keep the over-storage stock. Lee et al. (2016) develop an EOQ policy for a backlogged 
inventory system under deterministic demand, while Sana (2015) constructs a similar policy for a lost sale case under a series of demand distributions (including exponential, uniform, normal, log-normal, Poisson, and empirical). The lead time is neglected in both studies.

For periodic review, $(S, T)$ is a widely used policy. However, a study of the order-up-to level policy with storage space can only be found in Archibald (2007). The author considered an order-up-to level policy with a given review period for a single item in multiple locations. Transshipments between locations are allowed and the storage space capacity at each location represents the upper bound of the inventory on-hands. A rented warehouse is not allowed, implying that the order-up-to level is restricted by the storage capacity. The demand is modeled by the binomial distribution, replenishment lead time is not considered, and unsatisfied demand is backlogged. The optimal order-up-to level is determined by solving a Markov decision process.

To the best of our knowledge, there is no research that considers both the order-up-to level and the flexible storage capacity for non-perishable items. Therefore, we conduct such a study in this paper.

\section{Problem Statement}

In this study, a discrete-time inventory system with stochastic demand and lead time is considered. The demand $D$ in every period is assumed to be a discrete, independent, and identically distributed (i.i.d.) random variable with a probability mass function $f(d)$, where $d$ is a non-negative value of demand. In several practical situations, where the demand of many items does not follow most widely used theoretical distributions (Singha et al., 2017), $f(d)$ can be estimated by an empirical distribution. Similarly, the replenishment lead time $L$ is assumed to be i.i.d. with a probability mass function $f(l)$, where $l$ is a possible value of the lead time. In addition to the random demand and lead time, a periodic review, order-up-to level $(S, T)$ policy is employed in our system. The review period $T$ is assumed to be longer than the lead time (i.e., $T>L$ ). The inventory level is examined every $T$ periods and an order is placed with an ordering cost of $C_{P}$, to raise the inventory position to an order-up-to level $S$. The order is delivered after $L$ periods. Stockout may occur if the actual demand surpasses the inventory on-hands. Such a shortage is backlogged with a unit cost of $C_{S}$. To be representative of actual systems, we also consider two types of storage space in our inventory system. The first type, which has a fixed capacity of $W$ units, is regarded as an owned warehouse, and the second, whose capacity is unlimited, can be considered as a rented warehouse. When a replenishment order arrives, if the inventory level is above the capacity of the owned warehouse, the excessive units (over-storage units in this paper) are kept in the rented warehouse. There incurs a holding cost $C_{H}$ for every unit kept in the owned warehouses in every period, whereas a unit-time over-storage cost $C_{O} \geq C_{H}$ is charged for all items stored in the rented warehouse.

The objective is to determine the optimal policy parameters that minimize the total inventory management cost, including ordering, holding, over-storage, and shortage costs. Therefore, both order-up-to level $S$ and the review period $T$ are regarded as decision variables. According to Moharana and Sarmah (2016), this results in intractable formulations for an inventory system. To reduce the complexity, the review period $T$ is, at first, considered as a constant so that a mathematical model to determine the order-up-to level $S$ can be developed. After that, a heuristic procedure is constructed to search for the solutions of both $S$ and $T$.

The following notation is used in the remaining part of this paper.

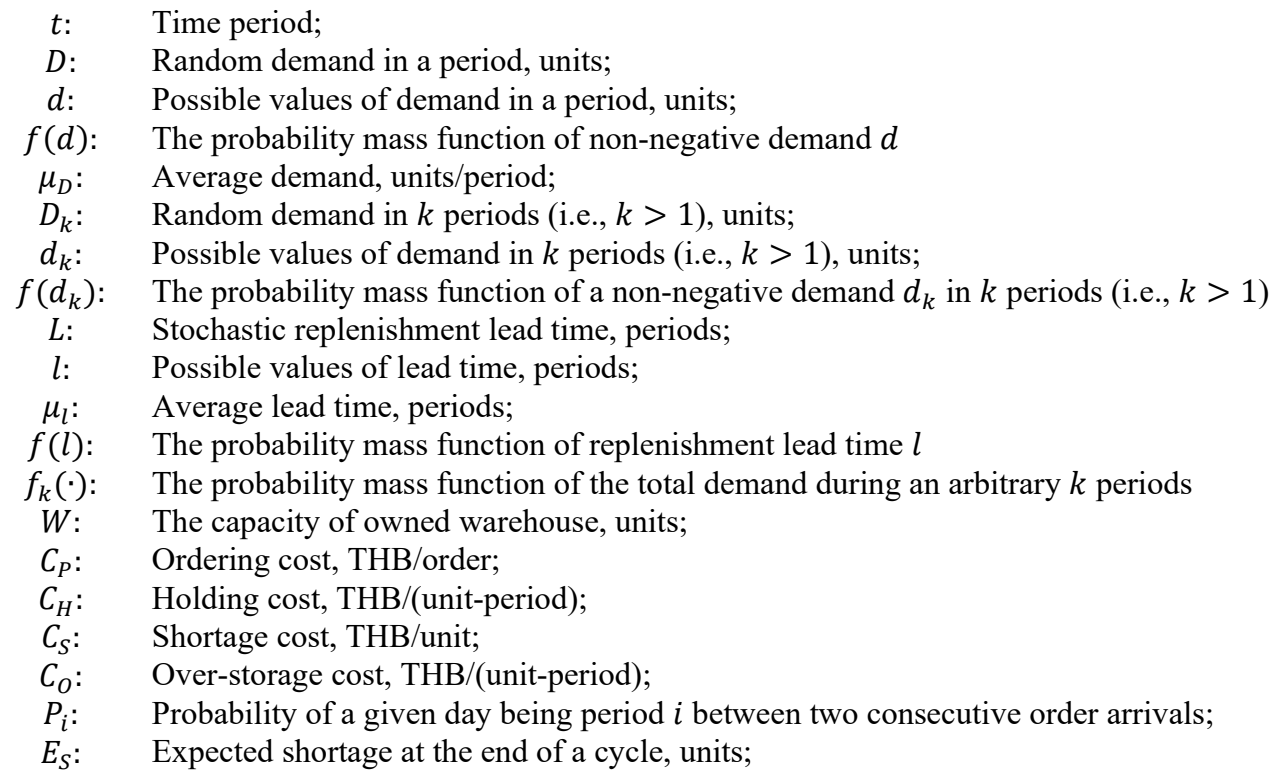


$P_{S}: \quad$ Probability of shortage at the end of a cycle, \%;

$E_{O}: \quad$ Expected over-storage, units;

$E_{O H}$ : Expected inventory on-hand in a cycle, units;

TC: Total inventory cost per cycle, THB/cycle;

\section{Model Development and Analysis}

\subsection{Demand during a protection interval}

According to Silver et al. (1998), an appropriate order-up-to level S must be large enough to cover the demand during a protection period (i.e., $L+T$ ) such that the total inventory cost over that period is minimal. Hence, it is necessary to model the total demand during $L+T$ periods before developing a total cost expression, associated with an order-up-to level $S$. With consideration of discrete random demand and lead time as introduced above, the probability of demand during a protection interval of $L+T$ periods can be determined by Eq. (1), using the method in Vollmann et al. (1997).

$$
f_{L+T}(x)=\sum_{l} P(X=x \mid L+T=l+T) \times P(L=l)
$$

where $X=x=d_{1}+d_{2}+\cdots+d_{l+T}=\sum_{k=1}^{l+T} d_{k}$ is a positive value of demand in $l+T$ periods, $d_{k} \in\left[d_{\text {min }}, d_{\text {max }}\right]$, and $P(X=x \mid L+T=l+T)=f\left(d_{1}\right) \times f\left(d_{2}\right) \times \cdots \times f\left(d_{l+T}\right)=\prod_{k=1}^{l+T} f\left(d_{k}\right)$ is the probability of demand $x$ in a given number of periods $l+T$.

\subsection{Expected shortage}

Based on the mass function $f_{L+T}(x)$, it is easy to compute the probability and the expected amount of shortage during a cycle, $L+T$. The probability of shortage $P_{S}$ and the expected shortage per cycle $E_{S}$ are expressed as:

$$
\begin{aligned}
& P_{S}=P(x>S)=\sum_{l} f_{L+T}(x), \\
& E_{S}=\sum_{x=S+1}^{x_{\max }}(x-S) f_{L+T}(x),
\end{aligned}
$$

\subsection{Mean-based estimation models}

Since we consider an inventory system with flexible storage capacity, it is necessary to measure the average amounts of inventory in the owned and rented warehouses. These values are known as the expected inventory on-hands $E_{O H}$ and overstorage $E_{O}$. The expressions for estimating these expectations are mathematically developed. Once they are obtained, the total cost function is constructed. This completes the model for the inventory system in our study. Traditionally, the expected inventory on-hands and over-storage can be estimated by using the mean-based approach (Hadley and Whitin, 1963). However, there are some known limitations of this approach when dealing with the discrete distributions of demand, lead time, and demand during protection interval in the case of a periodic review system. Therefore, this leads to less-accurate estimations of the expected inventory on-hands and over-storage. The shortcomings from the mean-based approach are overcome by using our novel period-based approach. To demonstrate this, it is worth to construct various models to estimate the expected on-hand inventory and over-storage from these approaches so that the performance of these estimations can be compared. Specifically, three models, two from the mean-based approach and one from the period-based approach, are developed. Each model is named corresponding to the approach, on which it is based, for ease of illustration. The development of these models is presented next.

\subsubsection{Mean-based (MB) model}

In this model, the expected on-hand inventory and over-storage are estimated by the mean demand and mean lead time. This mean-based approach is widely adopted in inventory research (Silver et al., 2016). The underlying assumption of this approach is that the inventory level in a system drops at a constant rate over time. Therefore, using the beginning and ending inventory levels is sufficient to estimate the average inventory level. By applying the approach, the estimations of expected inventory on-hands $E_{O H}^{M B}$ and over-storage $E_{O}^{M B}$ are expressed as follows.

$$
\begin{aligned}
& E_{O H}^{M B}=S-\mu_{D} \mu_{L}-\frac{\mu_{D} T}{2} \\
& E_{O}^{M B}=\Lambda
\end{aligned}
$$


where

$$
\begin{aligned}
& \Lambda=\left\{\begin{array}{cc}
\frac{\frac{1}{2}\left\{\max (0, \Gamma) \times \frac{\max (0, \Omega)}{\mu_{D}}\right\}}{T} & \text { if } \max (0, \Omega) / \mu_{D} \leq T, \\
S-\mu_{D} \mu_{L}-\frac{\mu_{D} T}{2}-W & \text { otherwise, }
\end{array}\right. \\
& \Gamma=S-W-\mu_{D} \mu_{L}, \text { and } \\
& \Omega=\Gamma+\mu_{D} .
\end{aligned}
$$

Eq. (4) is a common estimation for the expected inventory on-hands. This measurement usually represents the average amount of stock in a system, regardless of the location it is kept. In other words, this measure cannot capture the expected amount of stock in multiple locations as in the case of our study. Specifically, we consider an owned warehouse, in which inventory is prioritized to be placed, and a rented warehouse to keep the amount that is over the limit $W$ of the owned warehouse. Since keeping stocks at the rented warehouse is more expensive than at the owned house, it is necessary to estimate the over-storage inventory kept at the rented warehouse to minimize the total cost. By its nature, the over-storage amount is similar to inventory on-hands. However, since the stock in rented warehouse is always used first to satisfy demand, there are three cases that may occur: the over-storage depletes before, on, or after the review period $T$. In Eq. (5), the term $\max (0, \Gamma)$ represents the beginning level of over-storage at the time that a replenishment order arrives. In addition, the term $\max (0, \Omega) / \mu_{D}$ represents the number of periods it takes for the beginning over-storage amount to be completely depleted. If the over-storage amount becomes completely depleted on or before the next review period $T$, then $E_{O}^{M B}$ is computed by taking the beginning over-storage amount $\Gamma$, multiplying with $\max (0, \Omega) / \mu_{D}$ and dividing by 2 to compute the total amount of over-storage during the $\max (0, \Omega) / \mu_{D}$ periods, and then dividing by $T$ to compute the average amount per period. However, if $\Gamma$ is not depleted by the next review period, then the $E_{O}^{M B}$ is the average between $\Gamma$ and the remaining over-storage amount at time $T$. By using Eqs. (4) and (5), the total inventory cost function for the mean-based model is formulated as in the following equation.

$$
T C_{M B}=\frac{C_{P}}{T}+C_{H} \times E_{O H}^{M B}+\frac{C_{S} \times E_{S}}{T}+\left(C_{O}-C_{H}\right) \times E_{O}^{M B}
$$

\subsubsection{Extended mean-based (EMB) model}

In the MB model, only the average value of the lead time is used. However, there are situations where lead times vary within a few periods and often occur in integer values. In that case, a practical lead time distribution should be non-negative and discrete. This indicates a necessity to take these properties into account when estimating the average inventory on-hands and over-storage. To achieve that, an extended model, which is based on the MB model from the previous section, is developed. In the extended mean-based model, the estimations of the expected inventory on-hands $E_{O H}^{E M B}$ and over-storage $E_{O}^{M B}$ are presented in the following equations.

$$
\begin{aligned}
E_{O H}^{E M B} & =\sum_{l=l_{\min }}^{l_{\max }}\left(S-\mu_{D} \mu_{L}-\frac{\mu_{D} T}{2}\right) \times f(l), \text { and } \\
E_{O}^{E M B} & =\sum_{l=l_{\text {min }}}^{l_{\max }} \Upsilon \times f(l),
\end{aligned}
$$

where

$$
\begin{aligned}
& \Lambda=\left\{\begin{array}{cc}
\frac{\frac{1}{2}\left\{\max (0, \Xi) \times \frac{\max (0, \Theta)}{\mu_{D}}\right\}}{T} & \text { if } \max (0, \Theta) / \mu_{D} \leq T, \\
S-\mu_{D} l-\frac{\mu_{D} T}{2}-W & \text { otherwise, }
\end{array}\right. \\
& \Xi=S-W-\mu_{D} l, \text { and } \\
& \Theta=\Xi+\mu_{D} .
\end{aligned}
$$

Similar to the case of MB model, the total inventory cost function in this model is constructed by using Eqs. (7) and (8).

$$
T C_{E M B}=\frac{C_{P}}{T}+C_{H} \times E_{O H}^{E M B}+\frac{C_{S} \times E_{S}}{T}+\left(C_{O}-C_{H}\right) \times E_{O}^{E M B}
$$

\subsection{Period-based (PB) estimation model}

In the previous models, i.e. MB and EMB, the expected inventory on-hand has been determined by either $E_{O H}^{M B}=S-\mu_{D} \mu_{L}-$ $\mu_{D} T / 2$, which is the same as in the study of Hadley and Whitin (1963), or $E_{O H}^{E M B}=\sum_{l=l_{\text {min }}}^{l_{\max }}\left(S-\mu_{D} \mu_{L}-\mu_{D} T / 2\right) \times f(l)$, which accommodates a non-negative and discrete lead time distribution. However, these still might not be accurate estimations 
for a discrete inventory system that has a stochastic and highly variable demand. This is because the inventory of such a system does not deplete at a constant rate. Instead, the rate at which the inventory decreases varies significantly from period to period. Hence, relying on the beginning and ending levels of inventory on-hands may not be an appropriate approach. A similar argument is applied for the case of estimating expected over-storage. As a result, in order to measure these quantities accurately, a model is developed by employing a novel period-based approach. This period-based model is demonstrated by a numerical example that it can produce a better approximation than the other models from the mean-based approach.

\subsubsection{Expected inventory on-hands}

\section{The case of constant lead time}

To illustrate our period-based estimation approach, a simple case, where the lead time is constant, is presented first. After that, a general case, in which the lead time is stochastic, is discussed. For a simple case example, suppose $T=4$ and $L=2$, and consider two successive replenishment cycles, where two orders are placed and delivered.

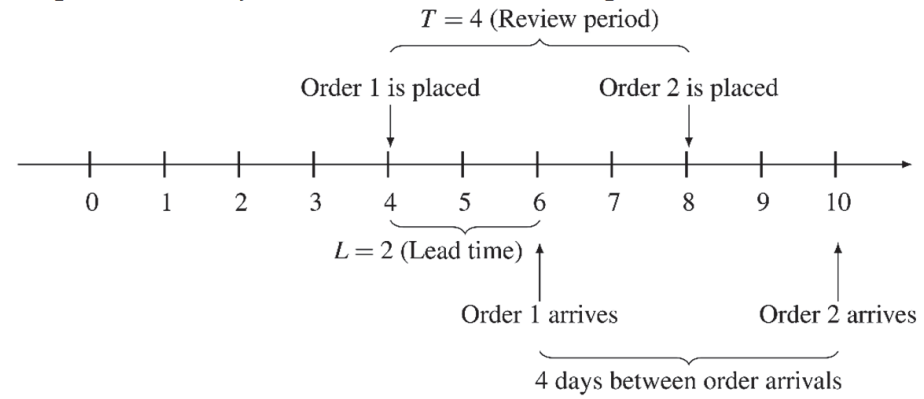

Fig. 1. Illustration of two successive order arrivals under deterministic lead time.

In Fig. 1, we, assume for simplicity of discussion that, a period is one day. Let $i$ be an index of days from Order 1 arrival to Order 2 arrival. With a constant lead time, there are 4 days between two orders, i.e., $i \in[1,4]$. That is, $i=1$ is the 1 st day at which Order 1 arrives (i.e., days of arrival), $i=2$ is the 2 nd day after Order 1 arrival, etc. Our approach will to estimate the expected inventory on-hand on each day $i$. With the probability $P(i)$ of any given day being day $i$ after an order arrives, we can estimate the expected inventory on-hand during a replenishment cycle. With $P_{i}=0.25 \forall i$, expected inventory on-hand for each day $i$ can be determined by using Eq. (10).

$$
E_{O H(i)}^{P B}=\sum_{y=0}^{S-1}(S-y) \times f_{L+i-1}(y)
$$

In Eq. (10), the expected inventory on-hand on day i, i.e., $O H(i)$, can be accurately estimated by determining the difference between $S$ and the random demand that occur during the $(L+i-1)$ days. For example, $O H(1)$ is the remaining inventory on-hand after satisfying the demand during lead time $L$. Similarly, $O H(2)$ is the remaining inventory on-hand after satisfying the demand during $L+1$ days.

\section{The case of random lead time}

Consider a case with stochastic lead time. The number of periods between two consecutive order arrivals, namely Order 1 arrival and Order 2 arrival, with lead time $L_{1}$ and $L_{2}$ are shown in Fig. 2. Between the two order arrivals, $i$ ranges from 1 to $T-L_{1}+L_{2}$ periods. Our approach estimates the expected inventory on-hand for each period $i \in\left[1, T-L_{1}+L_{2}\right]$ between the two order arrivals.

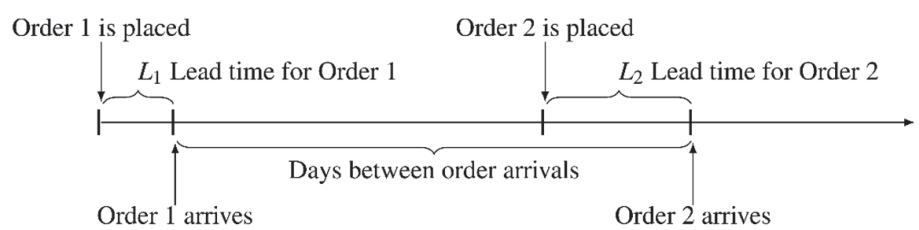

Fig. 2. Illustration of two successive order arrivals under stochastic lead time

For stochastic lead time, where $l \in\left[l_{\min }, l_{\max }\right]$, and $i \in\left[1, T-l_{\min }+l_{\max }\right]$, let $l_{1}$ and $l_{2}$ be the possible values of random variables $L_{1}$ and $L_{2}$, respectively. For a given day $i$, let $\left(l_{1}+i-1\right)$ denote the number of days between Order 1 placement and day $i$. The $O H(i)$ can be estimated by the difference between $S$ and the demand during $\left(l_{1}+i-1\right)$ days. At this point, for a given lead time $l_{1}$, let $P\left(l_{1}+i-1\right)$ denote the probability of having $\left(l_{1}+i-1\right)$ days (between Order 1 placement and day $i$ ). Therefore, $P\left(l_{1}+i-1\right)$ and $P_{i}$ can be determined by using Eq. (11) and Eq. (12) as follows.

$P\left(l_{1}+i-1\right)=\frac{A}{B}$, and 
$P_{i}=\frac{B}{T}$

where

$$
\begin{aligned}
A & =P\left(L_{1}=l_{1}\right) \times \sum_{l_{1}=\max \left(l_{\min }, i+l_{1}-T\right)}^{l_{\max }} P\left(L_{2}=l_{2}\right), \text { and } \\
\mathrm{B}=\sum_{l_{1}=l_{\text {min }}}^{\min \left(l_{\max }, T-i+l_{\max }\right)} & A .
\end{aligned}
$$

It should be noted that the term $A$ is calculated when $\max \left(1, i+l_{1}-T\right) \leq l_{\max }$. From Eq. (11), the probability of demand during $L_{1}+i-1$ days between Order 1 placement and day $i$ is calculated as:

$$
f_{L_{1}+i-1}(y)=\sum_{l=l_{\min }}^{l_{\max }} P\left(Y=y \mid l_{1}+i-1\right) \times P\left(l_{1}+i-1\right) \text {. }
$$

By using Eqs. (12) and (13), the expected inventory on-hand during the time after Order 1 arrives on day $L_{1}$ and before Order 2 arrives on day $T+L_{2}$ is expressed as:

$$
E_{O H}^{P B}=\sum_{i=1}^{T+l_{\max }-l_{\min }} E_{O H(i)}^{P B} \times P_{i}
$$

where

$$
E_{O H(i)}^{P B}=\sum_{y=0}^{S-1}(S-y) \times f_{L+i-1}(y) .
$$

To illustrate the suggested model, consider a numerical example in which the order-up-level $S=42$ units, the review period $T=4$ days, the demand follows a distribution in Table 1 , and lead times $L_{1}$ and $L_{2}$ follow a distribution as in Table 2.

Table 1

Distribution of demand

\begin{tabular}{lccccc}
\hline Demand & 0 & 1 & 2 & 4 & 6 \\
\hline Probability & 0.2 & 0.2 & 0.2 & 0.2 & 0.2 \\
\hline
\end{tabular}

Table 2

Distribution of lead time

\begin{tabular}{lccc}
\hline Lead time & 1 & 2 & 3 \\
\hline Probability & 0.7 & 0.2 & 0.1 \\
\hline
\end{tabular}

From Table 2, the possible maximum number of days after Order 1 arrival and before Order 2 arrival is $T-l_{\min }+l_{\max }=$ $4-1+3=6$ days. The actual number of days between Orders 1 and 2 arrivals depends on the values of $L_{1}$ and $L_{2}$. According to the distribution of lead time in Table 1, there are nine combinations of $L_{1}$ and $L_{2}$, which produce different numbers of days between the two order arrivals. These nine cases are illustrated in Fig. 3 .

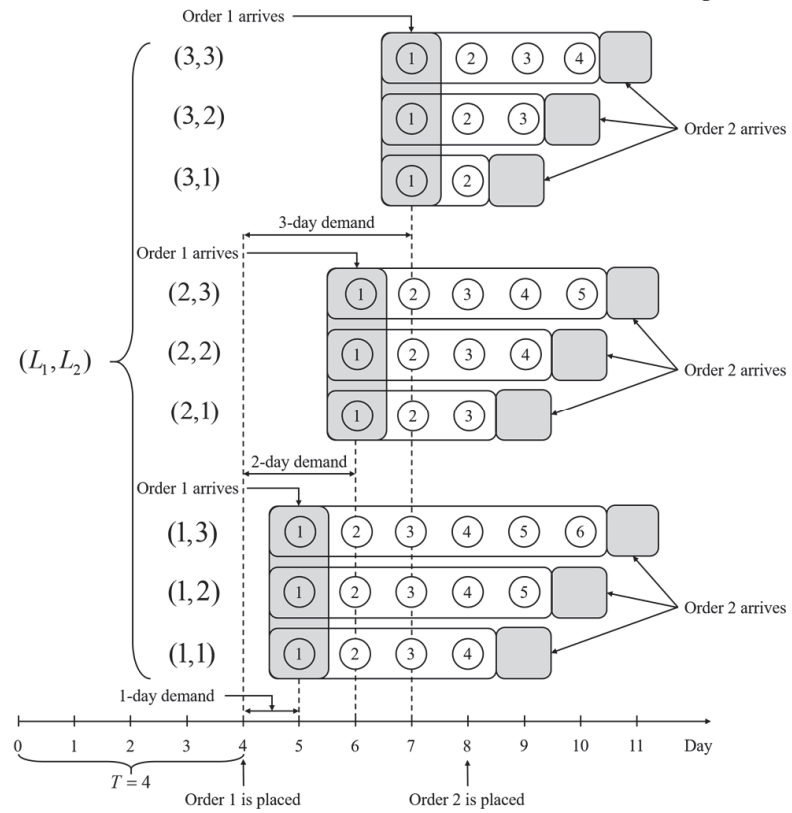

Fig. 3. Number of periods $(i)$ between Order 1 arrival and Order 2 arrival where $T=4$ and $L_{1}, L_{2} \in[1,2,3]$ 
Based on Table 2 and Fig. 3, the demand between ordering and the $i^{\text {th }}$ period after the order arrives is determined as follows.

- Demand between ordering and the $1^{\text {st }}$ period $(i=1)$ after the order arrives is the demand during a lead time of $\{1,2,3\}$ periods.

\begin{tabular}{|c|c|c|c|c|c|}
\hline$L_{1}=l_{1}$ & $L_{1}=l_{1}$ & Probability & $A$ & $l_{1}+i-1$ & $P\left(l_{1}+i-1\right)=A / B$ \\
\hline \multirow[t]{3}{*}{1} & 1 & $0.7 \times 0.7$ & 0.7 & 1 & $0.7 / 1=0.7$ \\
\hline & 2 & $0.7 \times 0.2$ & & & \\
\hline & 3 & $0.7 \times 0.1$ & & & \\
\hline \multirow[t]{3}{*}{2} & 1 & $0.2 \times 0.7$ & 0.2 & 2 & $0.2 / 1=0.2$ \\
\hline & 2 & $0.2 \times 0.2$ & & & \\
\hline & 3 & $0.2 \times 0.1$ & & & \\
\hline \multirow[t]{3}{*}{3} & 1 & $0.1 \times 0.7$ & 0.1 & 3 & $0.1 / 1=0.1$ \\
\hline & 2 & $0.1 \times 0.2$ & & & \\
\hline & 3 & $0.1 \times 0.1$ & & & \\
\hline \multicolumn{3}{|c|}{$B=0.7+0.2+0.1=1$} & & \multicolumn{2}{|c|}{$P_{1}=B / T=1 / 4=0.25$} \\
\hline
\end{tabular}

- $\quad$ Demand between ordering and the $2^{\text {nd }}$ period $(i=2)$ after the order arrives is the demand during (lead time +1$)$ periods, or $\{2,3,4\}$ periods.

\begin{tabular}{|c|c|c|c|c|c|}
\hline$L_{1}=l_{1}$ & $L_{1}=l_{1}$ & Probability & $A$ & $l_{1}+i-1$ & $P\left(l_{1}+i-1\right)=A / B$ \\
\hline \multirow[t]{3}{*}{1} & 1 & $0.7 \times 0.7$ & 0.7 & 2 & $0.7 / 1=0.7$ \\
\hline & 2 & $0.7 \times 0.2$ & & & \\
\hline & 3 & $0.7 \times 0.1$ & & & \\
\hline \multirow[t]{3}{*}{2} & 1 & $0.2 \times 0.7$ & 0.2 & 3 & $0.2 / 1=0.2$ \\
\hline & 2 & $0.2 \times 0.2$ & & & \\
\hline & 3 & $0.2 \times 0.1$ & & & \\
\hline \multirow[t]{3}{*}{3} & 1 & $0.1 \times 0.7$ & 0.1 & 4 & $0.1 / 1=0.1$ \\
\hline & 2 & $0.1 \times 0.2$ & & & \\
\hline & 3 & $0.1 \times 0.1$ & & & \\
\hline & $0.7+c$ & $+0.1=1$ & & $P_{1}=$ & $T=1 / 4=0.25$ \\
\hline
\end{tabular}

- Demand between ordering and the $3^{\text {rd }}$ period $(i=3)$ after the order arrives is the demand during (lead time +2$)$ periods, or $\{3, \underline{4,5}\}$ periods.

\begin{tabular}{|c|c|c|c|c|c|}
\hline$L_{1}=l_{1}$ & $L_{1}=l_{1}$ & Probability & $A$ & $l_{1}+i-1$ & $P\left(l_{1}+i-1\right)=A / B$ \\
\hline \multirow[t]{3}{*}{1} & 1 & $0.7 \times 0.7$ & 0.7 & 3 & $0.7 / 0.93=0.7$ \\
\hline & 2 & $0.7 \times 0.2$ & & & \\
\hline & 3 & $0.7 \times 0.1$ & & & \\
\hline \multirow[t]{3}{*}{2} & 1 & $0.2 \times 0.7$ & 0.2 & 4 & $0.2 / 0.93=0.2$ \\
\hline & 2 & $0.2 \times 0.2$ & & & \\
\hline & 3 & $0.2 \times 0.1$ & & & \\
\hline \multirow[t]{2}{*}{3} & 2 & $0.1 \times 0.2$ & 0.03 & 5 & $0.1 / 0.93=0.1$ \\
\hline & 3 & $0.1 \times 0.1$ & & & \\
\hline \multicolumn{4}{|c|}{$B=0.7+0.2+0.03=0.93$} & \multicolumn{2}{|c|}{$P_{1}=B / T=0.93 / 4=0.2325$} \\
\hline
\end{tabular}

- Demand between ordering and the $4^{\text {th }}$ period $(i=4)$ after the order arrives is the demand during (lead time +3$)$ periods, or $\{4,5,6\}$ periods.

\begin{tabular}{|c|c|c|c|c|c|}
\hline$L_{1}=l_{1}$ & $L_{1}=l_{1}$ & Probability & $A$ & $l_{1}+i-1$ & $P\left(l_{1}+i-1\right)=A / B$ \\
\hline \multirow[t]{3}{*}{1} & 1 & $0.7 \times 0.7$ & 0.7 & 4 & $0.7 / 0.77=0.9091$ \\
\hline & 2 & $0.7 \times 0.2$ & & & \\
\hline & 3 & $0.7 \times 0.1$ & & & \\
\hline \multirow[t]{2}{*}{2} & 2 & $0.2 \times 0.2$ & 0.06 & 5 & $0.06 / 0.77=0.0779$ \\
\hline & 3 & $0.2 \times 0.1$ & & & \\
\hline 3 & 3 & $0.1 \times 0.1$ & 0.01 & 6 & $0.01 / 0.77=0.0130$ \\
\hline \multicolumn{4}{|c|}{$B=0.7+0.06+0.01=0.77$} & \multicolumn{2}{|c|}{$P_{1}=B / T=0.77 / 4=0.1925$} \\
\hline
\end{tabular}

- Demand between ordering and the $5^{\text {th }}$ period $(i=5)$ after the order arrives is the demand during (lead time +4$)$ periods, or $\{5,6\}$ periods.

\begin{tabular}{cccccc}
\hline$L_{1}=l_{1}$ & $L_{1}=l_{1}$ & Probability & $A$ & $l_{1}+i-1$ & $P\left(l_{1}+i-1\right)=A / B$ \\
\hline 1 & 2 & $0.7 \times 0.2$ & 0.21 & 5 & $0.21 / 0.23=0.9130$ \\
2 & 3 & $0.7 \times 0.1$ & & & \\
2 & 3 & $0.2 \times 0.1$ & 0.02 & 6 & $0.02 / 0.23=0.0870$ \\
\hline \multicolumn{5}{c}{$B=0.21+0.02=0.23$} & \multicolumn{3}{c}{$P_{1}=B / T=0.23 / 4=0.0575$} \\
\hline
\end{tabular}


- Demand between ordering and the $6^{\text {th }}$ period $(i=6)$ after the order arrives is the demand during (lead time +5$)$ periods, or $\{6\}$ periods.

\begin{tabular}{|c|c|c|c|c|c|}
\hline$L_{1}=l_{1}$ & $L_{1}=l_{1}$ & Probability & $A$ & $l_{1}+i-1$ & $P\left(l_{1}+i-1\right)=A / B$ \\
\hline 1 & 3 & $0.7 \times 0.1$ & 0.07 & 6 & $0.07 / 0.07=1$ \\
\hline \multicolumn{4}{|c|}{$B=0.07$} & $P_{1}=B / 2$ & $=0.07 / 4=0.0175$ \\
\hline
\end{tabular}

With values of $P\left(l_{1}+i-1\right)$ as illustrated above, the distribution of demand between ordering and day $i$, i.e., $f_{L+i-1}(y)$, is obtained by using Eq. (13) and the expected inventory on-hand on day $i$ is calculated as $E_{O H(i)}^{P B}=\sum_{y=0}^{S-1}(S-y) \times f_{L+i-1}(y)$ : For example, the expected on-hand inventory on the $1^{\text {st }}$ day, i.e., $E_{O H(1)}$, is computed as follows.

\begin{tabular}{cccc}
\hline$y$ & $f_{3}(y)$ & $S-y$ & $(S-y) \times f_{3}(y)$ \\
\hline 0 & 0.1488 & 42 & 6.2496 \\
1 & 0.1584 & 41 & 6.4944 \\
2 & 0.1688 & 40 & 6.7520 \\
3 & 0.0216 & 39 & 0.8424 \\
4 & 0.1712 & 38 & 6.5056 \\
5 & 0.0232 & 37 & 0.8584 \\
6 & 0.1824 & 36 & 6.5664 \\
7 & 0.0256 & 35 & 0.8960 \\
8 & 0.0360 & 34 & 1.2240 \\
9 & 0.0072 & 33 & 0.2376 \\
10 & 0.0256 & 32 & 0.8192 \\
11 & 0.0048 & 31 & 0.1488 \\
12 & 0.0160 & 30 & 0.4800 \\
13 & 0.0024 & 29 & 0.0696 \\
14 & 0.0048 & 28 & 0.1344 \\
16 & 0.0024 & 26 & 0.0624 \\
18 & 0.0008 & 24 & 0.0192 \\
\hline
\end{tabular}

The expected inventory on-hand for other days, i.e., $E_{O H(i)} \forall i \in[2,6]$, are derived similarly. Once the expected inventory onhand of each day after an order arrives is determined, the expected inventory on-hand within a replenishment cycle is obtained by using Eq. (14).

\begin{tabular}{cccc}
\hline$i$ & $E_{O H(i)}^{P B}$ & $P_{i}$ & $E_{O H(i)}^{P B} \times P_{i}$ \\
\hline 1 & 38.3600 & 0.2500 & 9.5900 \\
2 & 35.7600 & 0.2500 & 8.9400 \\
3 & 33.4731 & 0.2325 & 7.7825 \\
4 & 31.3299 & 0.1925 & 6.0310 \\
5 & 28.7739 & 0.0575 & 1.6545 \\
6 & 26.4000 & 0.0175 & 0.4620 \\
\hline & & & 34.46 \\
\hline
\end{tabular}

\subsubsection{Expected over-storage}

Since there are two types of storage space in this system, estimating the expected amount of stock stored in the rented warehouse at the time of order arrival is necessary because of its contribution to the total inventory cost, which subsequently affects the optimal $S^{*}$. With the discrete demand during a protection interval of $L+T$ periods, the period-based approach, which is presented in the previous section, is employed to determine the expected over-storage amount. Similar to the case of the expected inventory on-hands, we also illustrate how the model from our period-based approach outperforms those from the mean-based approach, in the numerical experiments below.

By using the results from the approach to estimate the expected inventory on-hand, particularly, Eqs. (13) and (14), the expected over-storage is obtained as:

$$
E_{O}^{P B}=\sum_{i=1}^{T+l_{\max }-1} E_{O(i)}^{P B} \times P_{i}
$$

where 


$$
E_{O(i)}^{P B}=\sum_{y=0}^{S-W-1}(S-W-y) \times f_{L_{1}+i-1}(y) .
$$

\subsubsection{Total inventory cost}

From Eqs. (3), (7), and (9), the total inventory cost with regarding to backlogged is expressed as in the following equations.

$$
T C_{P B}=\frac{C_{P}}{T}+C_{H} \times E_{O H}^{P B}+\frac{C_{S} \times E_{S}}{T}+\left(C_{O}-C_{H}\right) \times E_{O}^{P B},
$$

By using Eq. (17), one can obtain the optimal values of the order-up-to level (i.e., $S^{*}$ ). This completes the period-based model. To obtain the solution for this model, a solution approach is developed and presented in the next section. It should be noted that this solution approach is also applicable to determine the solutions for both MB and EMB models.

\subsection{Solution approach}

Step 0: Determine the distribution of demand during protection interval $L+T$ by using Eq. (1). This distribution provides all possible values of demand $x \in\left[x_{\min }, x_{\max }\right]$ and their corresponding probability $f_{L+T}(x)$ :

Step 1: Set initial values for the review period, order-up-to level, and total cost as

$T^{*}=T=\operatorname{lmax}+1$

$S^{*}=S(T)=x_{\text {min }}$

$T C^{*}=T C(T)=M$,

where $M$ is a large number.

Step 2: Compute $P_{i}$ and the distribution of demand before period $i$, i.e., $f_{L+i-1}(x)$, by using Eqs. (12) and (13).

Step 3: Given $T, S(T)$ and other parameters, such as ordering $C_{P}$, holding $C_{H}$, shortage $C_{S}$, over-storage cost $C_{O}$, warehouse capacity $W$, the mass function of demand during protection interval $f_{L+T}(x)$, the probability of a period being period $i$ between two order arrivals $P_{i}$, and the mass function of demand before period $i$ between two consecutive orders $f_{L+i-1}(x)$, the total cost $T C(T)$ can be obtained by adding up all cost components, including ordering $C_{P} / T$, expected inventory on-hand $C_{H} \times E_{O H}$, expected shortage $\left(C_{S} \times E_{S}\right) / T$, and expected over-storage $C_{O} \times E_{O}$. These costs are determined by using Eqs. (3), (14), and (15).

Step 4: If $T C(T)<T C^{*}(T)$, set $T C^{*}(T)=T C(T), S^{*}(T)=S(T)$. If $S(T)<x_{\max }$, set $S(T)=S(T)+1$ and repeat Step 3 . Otherwise, go to Step 5.

Step 5: If $T C^{*}>T C^{*}(T)$, set $T C^{*}=T C^{*}(T), T^{*}=T, S^{*}=S^{*}(T), T=T+1, S(T)=x_{\min }$, and go to Step 3. Otherwise, stop the procedure.

\section{Computation Study}

In this section, a simulation model that mimics the behaviors of the $(S, T)$ inventory system is developed. The purpose is to compare the estimates of $E_{O H}, E_{O}$, and $T C$ from the three models, i.e., mean-based, extended mean-based, and period-based, with the simulation to assess their accuracy. The simulation was conducted with a replication length of 30,000 periods to ensure that the system behavior reaches its steady state. In the simulation, the ordering cost $C_{P}=0.2 \mathrm{THB} /$ order, the holding cost $C_{H}=0.0119 \mathrm{THB} /\left(\right.$ unit-period), and the over-storage cost $C_{O}$ (also in $\mathrm{THB} /($ unit-period)) is determined by the ratio of the over-storage cost to the holding cost $\left(C_{O} / C_{H}\right)$ in which $\left(C_{O} / C_{H}\right) \in[2,3, \ldots, 10]$ and the shortage cost $C_{S}=8 \mathrm{THB} /$ unit. The probability mass function of stochastic lead time is $f(l)=(1,0.7),(2,0.2),(3,0.1)$. The review period $T$ starts from 4 periods. In this simulation, we consider two types of product, slow- and fast-moving, where the data are obtained from the case study of a DC in Thailand. The properties of these two products are presented in Table 3.

Table 3

Characteristics of products

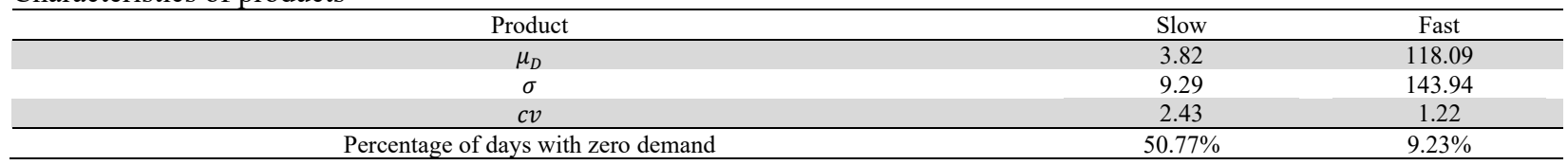


According to Table 3 , the average demand of the slow- and fast-moving products are 3.82 units/period and 118.09 units/period, respectively. The assigned capacity of the owned warehouse for the slow-moving product is 105 units and for the fast-moving product, 1,400 units. The computational results are summarized as follows.

Table 4

Performance of each model in comparison with simulation for the slow-moving product

\begin{tabular}{|c|c|c|c|c|c|c|c|}
\hline \multirow[b]{3}{*}{$T$} & \multirow[b]{3}{*}{$C_{O} / C_{H}$} & \multicolumn{6}{|c|}{ Model } \\
\hline & & \multicolumn{2}{|c|}{ MB } & \multicolumn{2}{|c|}{ EMB } & \multicolumn{2}{|c|}{$\mathrm{PB}$} \\
\hline & & $S_{M B}^{*}$ & $\Delta T C$ & $S_{E M B}^{*}$ & $\Delta T C$ & $S_{P B}^{*}$ & $\Delta T C$ \\
\hline \multirow{9}{*}{4} & 2 & 113 & $4.16 \%$ & 113 & $3.99 \%$ & 111 & $0.46 \%$ \\
\hline & 3 & 112 & $5.74 \%$ & 112 & $5.39 \%$ & 109 & $0.43 \%$ \\
\hline & 4 & 111 & $6.75 \%$ & 111 & $6.25 \%$ & 108 & $0.41 \%$ \\
\hline & 5 & 111 & $8.33 \%$ & 110 & $6.63 \%$ & 107 & $0.39 \%$ \\
\hline & 6 & 111 & $9.91 \%$ & 110 & $7.79 \%$ & 106 & $0.38 \%$ \\
\hline & 7 & 111 & $11.49 \%$ & 110 & $8.95 \%$ & 106 & $0.38 \%$ \\
\hline & 8 & 110 & $10.91 \%$ & 109 & $8.33 \%$ & 105 & $0.35 \%$ \\
\hline & 9 & 110 & $12.19 \%$ & 109 & $9.24 \%$ & 105 & $0.35 \%$ \\
\hline & 10 & 110 & $13.47 \%$ & 109 & $10.15 \%$ & 105 & $0.35 \%$ \\
\hline \multirow{9}{*}{5} & 2 & 118 & $6.37 \%$ & 115 & $4.10 \%$ & 114 & $0.52 \%$ \\
\hline & 3 & 114 & $6.07 \%$ & 114 & $5.79 \%$ & 111 & $0.60 \%$ \\
\hline & 4 & 113 & $7.45 \%$ & 113 & $7.04 \%$ & 109 & $0.64 \%$ \\
\hline & 5 & 112 & $8.33 \%$ & 112 & $7.78 \%$ & 108 & $0.66 \%$ \\
\hline & 6 & 111 & $8.69 \%$ & 111 & $8.03 \%$ & 107 & $0.67 \%$ \\
\hline & 7 & 111 & $10.00 \%$ & 111 & $9.20 \%$ & 107 & $0.67 \%$ \\
\hline & 8 & 111 & $11.31 \%$ & 110 & $8.84 \%$ & 106 & $0.70 \%$ \\
\hline & 9 & 111 & $12.61 \%$ & 110 & $9.79 \%$ & 106 & $0.70 \%$ \\
\hline & 10 & 111 & $13.92 \%$ & 110 & $10.74 \%$ & 106 & $0.70 \%$ \\
\hline
\end{tabular}

Note: $\triangle T C$ is the percentage difference in the total cost between each model and the simulation at the corresponding optimal solution.

Table 5

Performance of each model in comparison with simulation for the fast-moving product

\begin{tabular}{|c|c|c|c|c|c|c|c|}
\hline \multirow[b]{3}{*}{$T$} & \multirow[b]{3}{*}{$C_{O} / C_{H}$} & \multicolumn{6}{|c|}{ Model } \\
\hline & & \multicolumn{2}{|c|}{ MB } & \multicolumn{2}{|c|}{ EMB } & \multicolumn{2}{|c|}{ PB } \\
\hline & & $S_{M B}^{*}$ & $\Delta T C$ & $S_{E M B}^{*}$ & $\Delta T C$ & $S_{P B}^{*}$ & $\Delta T C$ \\
\hline \multirow{9}{*}{4} & 2 & 1697 & $7.22 \%$ & 1697 & $6.76 \%$ & 1680 & $0.28 \%$ \\
\hline & 3 & 1661 & $9.58 \%$ & 1660 & $8.67 \%$ & 1634 & $0.23 \%$ \\
\hline & 4 & 1639 & $11.62 \%$ & 1637 & $10.26 \%$ & 1602 & $0.18 \%$ \\
\hline & 5 & 1622 & $13.38 \%$ & 1623 & $11.66 \%$ & 1579 & $0.14 \%$ \\
\hline & 6 & 1610 & $15.03 \%$ & 1609 & $12.76 \%$ & 1562 & $0.13 \%$ \\
\hline & 7 & 1600 & $16.54 \%$ & 1597 & $13.71 \%$ & 1547 & $0.12 \%$ \\
\hline & 8 & 1592 & $17.99 \%$ & 1587 & $14.57 \%$ & 1536 & $0.11 \%$ \\
\hline & 9 & 1586 & $19.42 \%$ & 1579 & $15.39 \%$ & 1525 & $0.11 \%$ \\
\hline & 10 & 1580 & $20.72 \%$ & 1571 & $16.08 \%$ & 1516 & $0.10 \%$ \\
\hline \multirow{9}{*}{5} & 2 & 1815 & $7.31 \%$ & 1815 & $6.99 \%$ & 1805 & $0.62 \%$ \\
\hline & 3 & 1766 & $9.55 \%$ & 1766 & $8.93 \%$ & 1747 & $0.44 \%$ \\
\hline & 4 & 1734 & $11.43 \%$ & 1736 & $10.53 \%$ & 1707 & $0.27 \%$ \\
\hline & 5 & 1711 & $13.07 \%$ & 1711 & $11.79 \%$ & 1678 & $0.13 \%$ \\
\hline & 6 & 1692 & $14.05 \%$ & 1692 & $12.90 \%$ & 1655 & $0.01 \%$ \\
\hline & 7 & 1678 & $15.85 \%$ & 1677 & $13.90 \%$ & 1637 & $0.09 \%$ \\
\hline & 8 & 1665 & $17.03 \%$ & 1664 & $14.78 \%$ & 1621 & $0.16 \%$ \\
\hline & 9 & 1655 & $18.21 \%$ & 1653 & $15.61 \%$ & 1608 & $0.22 \%$ \\
\hline & 10 & 1646 & $19.30 \%$ & 1643 & $16.34 \%$ & 1596 & $0.27 \%$ \\
\hline
\end{tabular}

Note: $\triangle T C$ is the percentage difference in the total cost between each model and the simulation at the corresponding optimal solution.

As can be seen in Tables 4 and 5, we compare the total inventory costs obtained from the three models, including MB, EMB, and $\mathrm{PB}$, with the total costs from the simulation of the $(S, T)$ inventory system for the slow- and fast-moving products, respectively. The PB model outperforms the MB and EMB models in terms of $\triangle T C$ in all instances. In addition, the EMB model is slightly better than the MB model with respect to $\triangle T C$. Moreover, it is realized that PB model is effective at all tested values of the ratio of over-storage $\operatorname{cost} C_{O}$ to the inventory holding $\operatorname{cost} C_{H}$, i.e., $\triangle T C$ is less than $1 \%$ in all cases. The $\Delta T C$ for the MB and EMB models become larger as $C_{O} / C_{H}$ increases. When the ratio $C_{O} / C_{H}$ increases from 2 to 10 , the optimal value $S_{P B}^{*}$ of the PB model decreases. On the other hand, the optimal values $S_{M B}^{*}$ and $S_{E M B}^{*}$ of the MB and EMB models decrease and then remain constant for most values of $C_{O} / C_{H}$ in the case of slow moving product, while they decrease and always stay above the optimal value $S_{P B}^{*}$ of the PB model in the case of fast-moving product.

The gap in the performance and the difference in the optimal $S^{*}$ values among the three models can be attributed to the difference in how they estimate the expected inventory on-hands and over-storage amounts. From Fig. 4, for the slow-moving product with a review period $T=5$, there is a difference in the expected inventory on-hand and the expected over-storage among the MB, EMB, and PB models. Fig 4a. illustrates that the MB and EMB models (the dashed and dash-dotted line, 
respectively) always under-estimate the expected inventory on-hand, compared to the PB model (the solid line), whose results are more consistent with the simulation results.

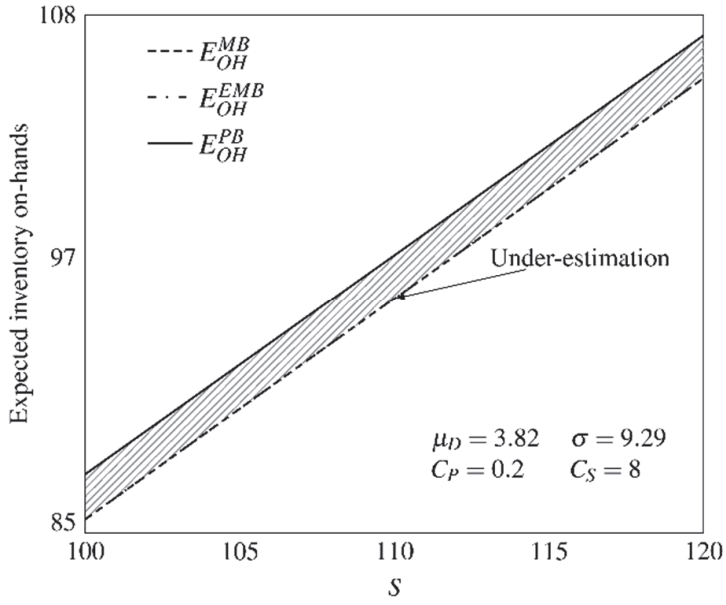

(a) Expected inventory on-hands

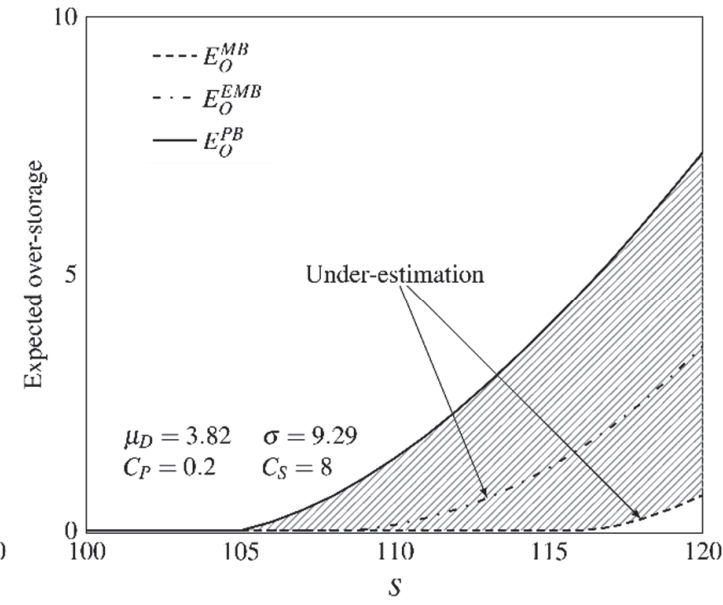

(b) Expected over-storage

Fig. 4. Comparison of $E_{O H}$ and $E_{O}$ for slow-moving product with $T=5$.

Similarly, Fig. 4b demonstrates that when order-up-to level S increases from 100 to 120, the expected over-storage is also under-estimated by the MB and EMB models. The underestimation leads to the differences in the total cost and the optimal $S^{*}$ of the three models, as seen in Fig. 5. In other words, because of the under-estimation, both the MB and EMB models estimate that $E_{O}$ has already reached zero at higher values of the order-up-to level $S$ (sub-optimal) solution.

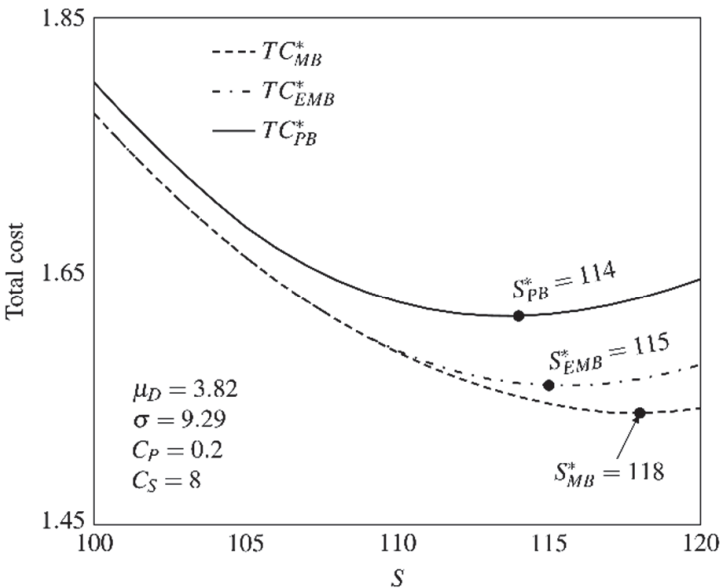

(a) $C_{O} / C_{H}=2$

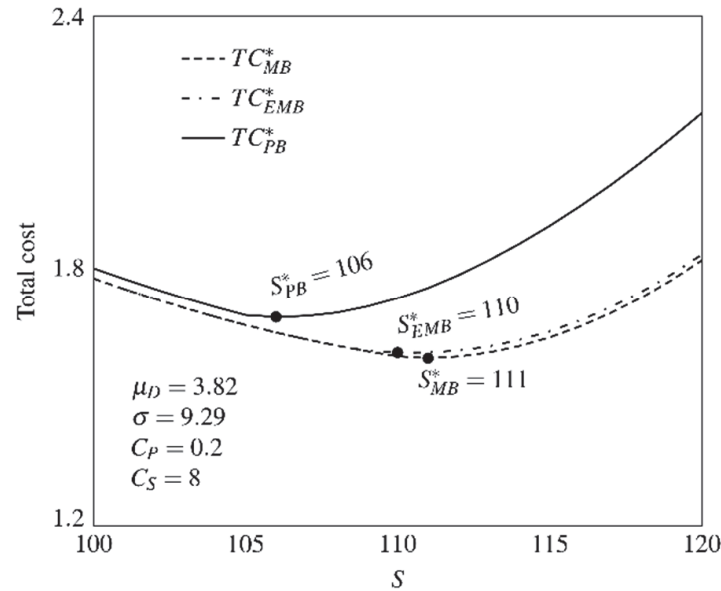

(b) $C_{O} / C_{H}=8$

Fig. 5. Total inventory cost for slow-moving product with $T=5$

When the ratio of the over-storage cost to the inventory on-hand cost is low (i.e., $C_{O} / C_{H}=2$ ), the MB, EMB, and PB models produce optimal $S^{*}$ values of 118,115 , and 114 , respectively (See Fig. 5a). In contrast, when the cost ratio is large (i.e., $\left.C_{O} / C_{H}=8\right)$, the optimal $S^{*}$ values are 111 for the MB model, 110 for EMB model, and 106 for the PB model (See Fig. 5b). These observations can be explained as follows.

When the over-storage cost is not expensive, the three models keep some stocks in the external warehouse, which incurs an over-storage cost. However, this can be compensated for by the savings from a lower shortage cost due to more available stock to satisfy customer demand, which results in larger values of S. From Fig. $4 \mathrm{~b}$, at $S>114$, the MB and EMB models underestimate the amount of expected over-storage, compared to the PB model. This leads to the gaps of the total costs between the MB, EMB models and the simulation, because there are actually larger amounts of over-storage cost in the simulation result than that in the MB and EMB solutions. In other words, the benefit of having more stock in the external warehouse is undermined in the MB and EMB models. Hence, these models keep the amount of stock to a level that is higher than that of the PB model (i.e., $S_{M B}^{*}>S_{E M B}^{*}>S_{P B}^{*}$ ). With an expensive over-storage cost, all models avoid having stocks in the external warehouse, which incurs an enormous expense. This expense cannot be recovered by the savings from the reduced shortage cost. That is, the three models attempt to lower the order-up-to level $\mathrm{S}$ to avoid over-storage. From Fig. $4 \mathrm{~b}$, the MB and EMB models estimate that over-storage would not have occurred when $S$ is lower than 111 and 110 , respectively. This is 
the reason why their optimal $S_{M B}^{*}$ and $S_{E M B}^{*}$ values are 111 and 110, respectively. The PB model, in contrast, shows that overstorage still occurs with $S$ values lower than 110. The PB model indicates that over-storage would not occur when $S$ is below 106. As a result, its optimal $S_{P B}^{*}$ is lower at 106 .

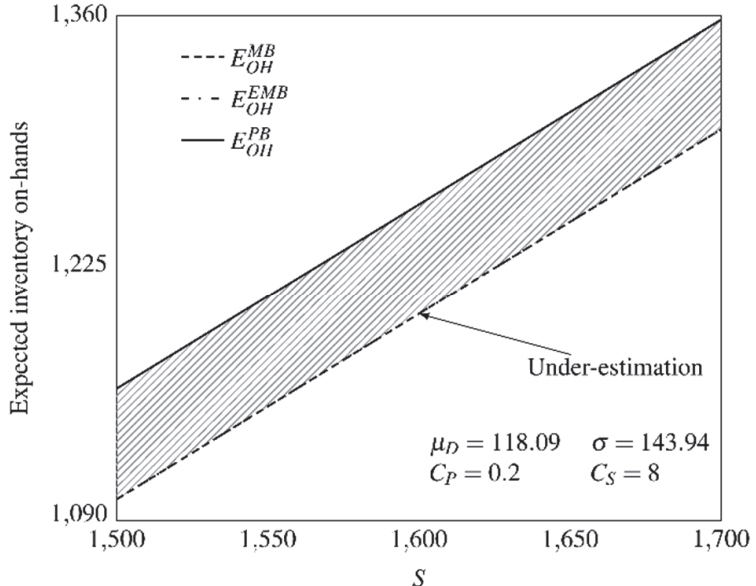

(a) Expected inventory on-hands

Fig. 6. Comparison of $E_{O H}$ and $E_{O}$ for fast-moving product with $T=4$.

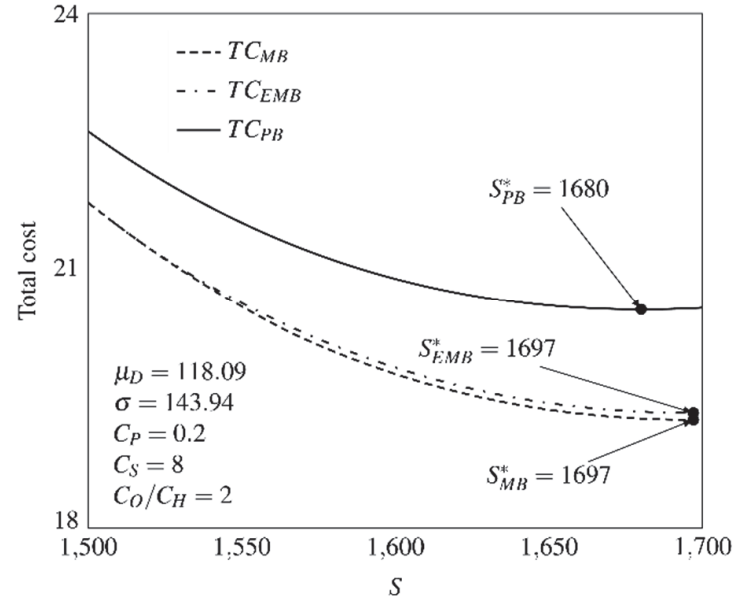

(a) $C_{O} / C_{H}=2$

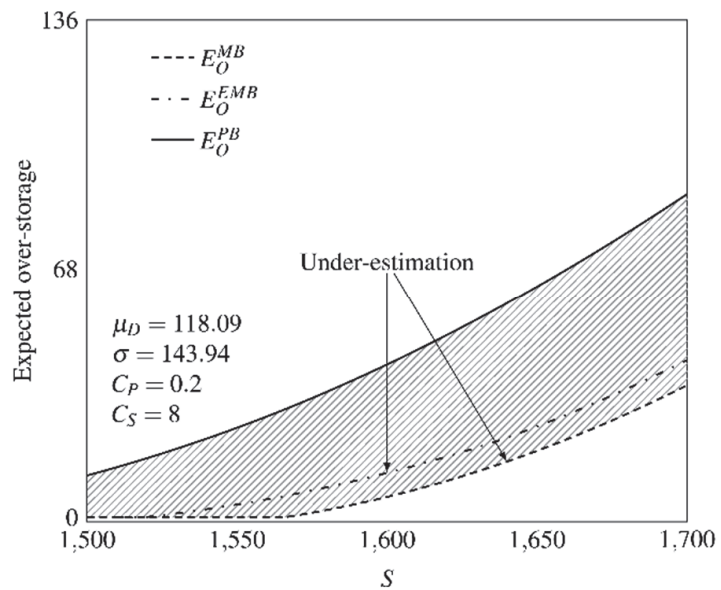

(b) Expected over-storage

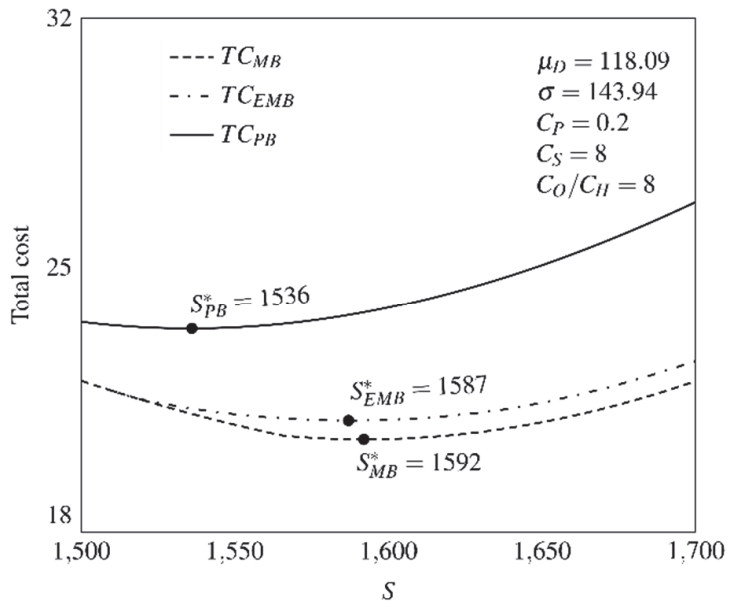

(b) $C_{O} / C_{H}=8$

Fig. 7. Total inventory cost for fast-moving product with $T=4$.

The results for the fast-moving product in Figs. 6 and 7 can be interpreted in a similar fashion. The results from the three models clearly show that the PB model provides better estimates than the MB and EMB models for the system under study. In other words, the inventory model developed by our proposed period-based approach outperforms those obtained from the mean-based approach. This is attributed to the non-negative and discrete properties of distributions for demand, lead time, and demand during protection interval in the case of periodic review system.

\section{Conclusion}

In this paper, a new period-based approach for a $(S, T)$ capacitated inventory system with backlog under stochastic discrete demand and lead time has been proposed. Based on this approach, a mathematical model, namely period-based model, that can accurately estimate the system performance has been developed. By using this model, the optimal review period and order-up-to level are determined. The effectiveness of the period-based model is compared with two other models, which are constructed by using the mean-based approach. Through a computational study, based on real datasets of slow- and fastmoving products, it is demonstrated that the model from our period-based approach outperforms those from the mean-based approach. Our model can accurately estimate the expected inventory on-hands and over-storage amounts, while the other models underestimates these values. With the results of this research, our period-based approach can be extended to accommodate other widely-adopted inventory policies such as $(Q, R)$ and $(s, S)$. In addition, it would be interesting to apply this approach for a lost sale system and examine its performance with various inventory policies.

\section{References}

Archibald, T. W. (2007). Modelling replenishment and transshipment decisions in periodic review multilocation inventory systems. Journal of the Operational Research Society, 58(7), 948-956. 
Bakker, M., Riezebos, J., \& Teunter, R. H. (2012). Review of inventory systems with deterioration since 2001. European Journal of Operational Research, 221(2), 275-284.

Bulinskaya, E. V. (1990). Inventory control in case of unknown demand distribution. Engineering Costs and Production Economics, 19(1-3), 301-306.

Gallego, G. (1992). A minmax distribution free procedure for the (Q, R) inventory model. Operations Research Letters, 11(1), $55-60$.

Gallego, G., \& Moon, I. (1993). The distribution free newsboy problem: review and extensions. Journal of the Operational Research Society, 44(8), 825-834.

Hadley, G.. \& Whitin, T. M. (1963). Analysis of Inventory Systems. Prentice-Hall.

Hariga, M. A. (2010). A single-item continuous review inventory problem with space restriction. International Journal of Production Economics, 128(1), 153-158.

Janssen, L., Claus, T., \& Sauer, J. (2016). Literature review of deteriorating inventory models by key topics from 2012 to 2015. International Journal of Production Economics, 182, 86-112.

Keaton, M. (1995). Using the gamma distribution to model demand when lead time. Journal of Business Logistics, $16(1), 107$.

Lee, H. L., Padmanabhan, V., \& Whang, S. (1997a). The bullwhip effect in supply chains. Sloan Management Review, 38, 93-102.

Lee, H. L., Padmanabhan, V., \& Whang, S. (1997b). Information distortion in a supply chain: The bullwhip effect. Management science, 43(4), 546-558.

Lee, J. Y., Cho, R. K., \& Paik, S. K. (2016). Supply chain coordination in vendor-managed inventory systems with stockoutcost sharing under limited storage capacity. European Journal of Operational Research, 248(1), 95-106.

Levén, E., \& Segerstedt, A. (2004). Inventory control with a modified Croston procedure and Erlang distribution. International journal of production economics, 90(3), 361-367.

Moharana, U. C., \& Sarmah, S. P. (2016). Determination of optimal order-up to level quantities for dependent spare parts using data mining. Computers \& Industrial Engineering, 95, 27-40.

Moon, I., \& Choi, S. (1994). The distribution free continuous review inventory system with a service level constraint. Computers \& industrial engineering, 27(1-4), 209-212.

Moon, I., \& Choi, S. (1995). The distribution free newsboy problem with balking. Journal of the Operational Research Society, 46(4), 537-542.

Nenes, G., Panagiotidou, S., \& Tagaras, G. (2010). Inventory management of multiple items with irregular demand: A case study. European Journal of Operational Research, 205(2), 313-324.

Pan, A., Hui, C. L., \& Ng, F. (2014). An optimization of inventory policy based on health care apparel products with compound poisson demands. Mathematical Problems in Engineering, 2014.

Sana, S. S. (2015). An EOQ model for stochastic demand for limited capacity of own warehouse. Annals of Operations Research, 233(1), 383-399.

Scarf, H. (1958). A min-max solution of an inventory problem. Studies in the Mathematical Theory of Inventory and Production.

Silver, E. A., Pyke, D. F., \& Peterson, R. (1998). Inventory management and production planning and scheduling (Vol. 3, p. 30). New York: Wiley.

Silver, E. A., Pyke, D. F., \& Thomas, D. J. (2016). Inventory and production management in supply chains. CRC Press.

Singha, K., Buddhakulsomsiri, J., \& Parthanadee, P. (2017). Mathematical Model of Inventory Policy under Limited Storage Space for Continuous and Periodic Review Policies with Backlog and Lost Sales. Mathematical Problems in Engineering, 2017.

Singha, K., Buddhakulsomsiri, J., \& Parthanadee, P. (2019). Computational experiment of methods to determine periodic (R, Q) inventory policy parameters: a case study of information decentralised distribution network. International Journal of Industrial and Systems Engineering, 32(2), 212-242.

Teunter, R. H., Syntetos, A. A., \& Babai, M. Z. (2010). Determining order-up-to levels under periodic review for compound binomial (intermittent) demand. European Journal of Operational Research, 203(3), 619-624.

Vollmann, T., Berry, W., and Whybark, D. (1997). Manufacturing Planning and Control System. Irwin/McGraw-Hill: New York, 4th edition.

Zhao, X., Qiu, M., Xie, J., \& He, Q. (2012). Computing (r, Q) policy for an inventory system with limited sharable resource. Computers \& Operations Research, 39(10), 2368-2379.

Zipkin, P. (1988). The use of phase-type distributions in inventory-control models. Naval Research Logistics (NRL), 35(2), 247-257.

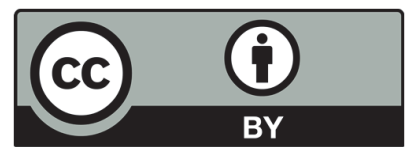

(C) 2021 by the authors; licensee Growing Science, Canada. This is an open access article distributed under the terms and conditions of the Creative Commons Attribution (CCBY) license (http://creativecommons.org/licenses/by/4.0/). 